Utah State University

DigitalCommons@USU

$1-1-1998$

\title{
Optical Stability Testing of the Fiber Support Technology (FiST) Focal Plane Assembly of the SABER Instrument
}

Scott M. Jensen

J. Clair Batty

Roy Esplin

Matthew J. Felt

Follow this and additional works at: https://digitalcommons.usu.edu/sdl_pubs

\section{Recommended Citation}

Jensen, Scott M.; Batty, J. Clair; Esplin, Roy; and Felt, Matthew J., "Optical Stability Testing of the Fiber Support Technology (FiST) Focal Plane Assembly of the SABER Instrument" (1998). Space Dynamics Lab Publications. Paper 62.

https://digitalcommons.usu.edu/sdl_pubs/62

This Article is brought to you for free and open access by the Space Dynamics Lab at DigitalCommons@USU. It has been accepted for inclusion in Space Dynamics Lab Publications by an authorized administrator of DigitalCommons@USU. For more information, please contact digitalcommons@usu.edu.

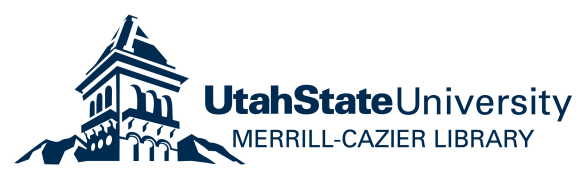




\title{
Optical stability testing of the Fiber Support Technology (FiST) focal plane assembly of the SABER instrument
}

\author{
Scott M. Jensen ${ }^{2}$, J. Clair Batty ${ }^{\mathbf{a}}$, Roy Esplin', and Matthew J. Felt ${ }^{\mathbf{a}}$ \\ ${ }^{a}$ Space Dynamics Laboratory, Utah State University \\ 1695 North Research Park Way, Logan, Utah 84341
}

\begin{abstract}
The focal plane assembly of the Sounding of the Atmosphere using Broadband Emission Radiometry (SABER) instrument is supported using Fiber Support Technology (FiST) which utilizes high performance fibers in tension to mechanically support and thermally isolate a cooled component from a warm environment. Details of this approach were presented in detail at SPIE meeting in Denver in 1996. The SABER team deemed it necessary to perform optical stability testing on this never-beforeflown technology for supporting focal plane assemblies to determine if precise positioning could be maintained through vibration and thermal cycling.
\end{abstract}

After subjecting the support system to vibration and thermal cycling, the angular orientation between the warm outer support structure and the inner cold block was measured. Since the outer support structure serves as the reference location for positioning the focal plane assembly and the cold block is where the detectors reside, it was possible to determine if FiST meets the optical stability requirements for the SABER instrument.

The results from this testing are presented, discussed, and compared to the optical requirements of the SABER instrument. A brief summary of current thermal and mechanical enhancements to the system will also be discussed.

Keywords: SABER, FiST, Stability, Tension, Fiber

\section{INTRODUCTION}

A major challenge faced by those developing cryogenically cooled components for space applications is to provide both adequate mechanical support and thermal isolation. If the cryogenic system also includes an optical device, a third requirement of optical stability is also needed. Depending on the system, the stability requirement may be easily achieved or, as was the case for the Sounding of the Atmosphere using Broadband Emission Radiometry (SABER) instrument, more difficult to achieve. The SABER requires that the focal plane assembly (FPA) be within a 60 arc-second envelope at operational temperatures for the lifetime of the mission.

Traditionally, concentric composite G-10 cylinders (See Figure 1) have supported cold components developed by Utah State University/Space Dynamics Laboratory (USU/SDL). While this approach has been accepted to be optically stable, it still has two limitations that are becoming more serious in the new "smaller, faster, cheaper, better" attitude of the 90's. 


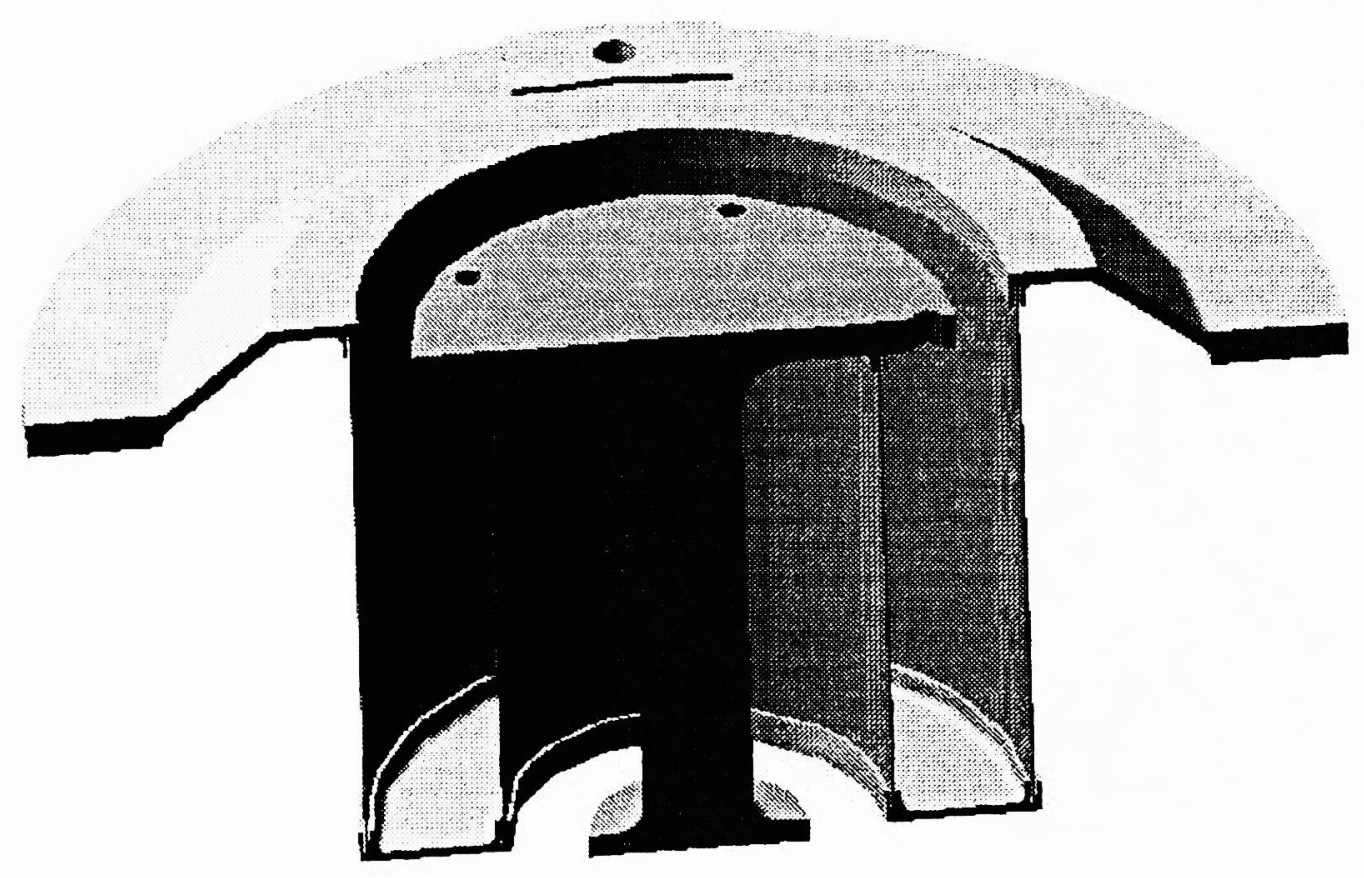

Figure 1. Section View of a Traditional G-10 support approach.

First, the parasitic heat loads due to conduction through this type of system have a significant impact on cooling capacity. For systems using expendable cryogen as the heat sink, mission life is limited. In systems using mechanical coolers, the cooling capacity may be exceeded. Second, the first natural resonant frequency of systems similar to that depicted in Figure $l$ is dangerously close to the response felt during launch. Higher values are desirable to avoid the risk of resonant response.

Cooling of the infrared sensors on the SABER instrument is provided by a TRW miniature pulse tube refrigerator, which provides roughly $250 \mathrm{~mW}$ of cooling at a cold block temperature of $72 \mathrm{~K}$ and 20 watts input power to the compressor'. The SABER FPA, if connected to this support scheme, would dissipate more heat than the refrigerator has capacity to cool, making it impossible to reach the desired detector temperature of $72 \mathrm{~K}$. This problem would preclude the use of this small, lightweight, low input power refrigerator. The SABER instrument would then not be possible under mass and power constraints imposed by the spacecraft.

Fiber Support Technology (FiST) was developed to significantly reduce parasitic heat loads, which in turn makes possible the use of miniature mechanical refrigerators as a cooling option. This system utilizes high performance fibers (in this case Kevlar) in tension to thermally isolate and mechanically support the cooled detector assembly. This approach is depicted in Figure 2. The thermal and mechanical details of this approach were presented at the Denver SPIE conference in 1996 and will not be discussed here ${ }^{2}$. However, a brief overview of thermal and mechanical performance, and some of the changes made to the system since that time will be mentioned.

FiST has two advantages that have been proven over significant breadboard testing. First, it is thermally superior to the standard G-10 approach. For the SABER FPA, measured FPA heat loads in the hot case are approximately $350 \mathrm{~mW}$ using the FiST support approach and $500 \mathrm{~mW}$ using the G-10 support approach. Parasitic heat loads due to conduction account for most of the difference but radiation parasitics are also larger in the G-10 approach due to the increased cold surface area. Second, FiST is mechanically robust. The first natural resonant frequency of the FiST system is has been measured to be nearly $800 \mathrm{~Hz}$ the G-10 approach has a first natural resonant frequency of approximately $180 \mathrm{~Hz}$. The added stiffiess of the FiST system reduces the risk of coupling with instrument/spacecraft frequencies during launch. 
Because this is a new technology, many questions concerning the long-term optical stability of the system were posed. The SABER FPA will be thermally cycled from room temperature down to $72 \mathrm{~K}$ many times over the life of the instrument. Since maintaining precise position of the detector assembly over the lifetime of the mission is a must, a significant test effort was established to determine if FiST was optically stable and if not how to correct it. The following sections of this report will discuss this effort.
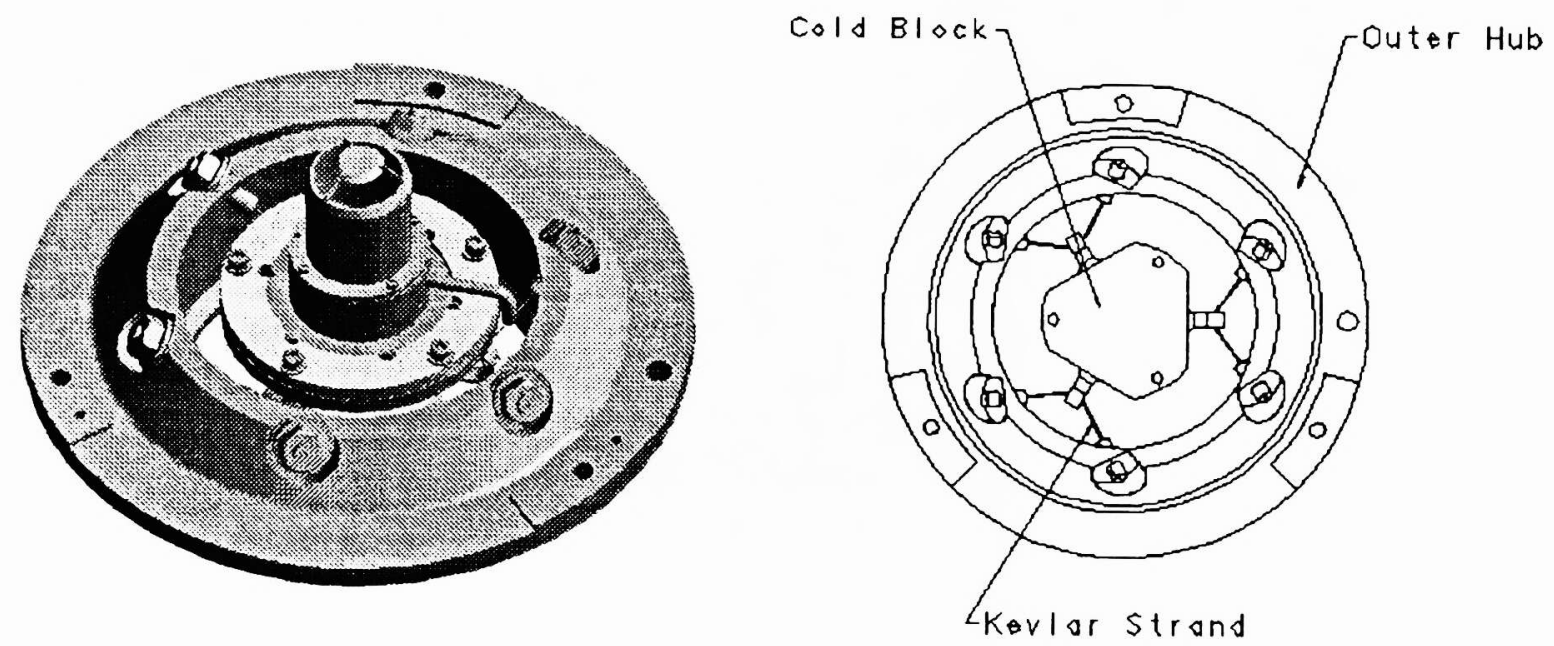

Figure 2. Fiber Support Technology (FiST)

\section{OPTICAL STABILITY}

FiST posses wonderful thermal and mechanical properties that make it ideal for use as a focal plane support. These characteristics cannot be taken advantage of if optical stability is not achieved. The requirements for SABER specify that the angular orientation of the focal plane with respect to the outer hub of FiST must be within a 60 arc-second envelope during operation throughout the mission. This corresponds to a 0.00022 inch change in height of the outer edge of the focal plane assuming that the center point of the focal plane is fixed. That's about $1 / 20^{\text {th }}$ the thickness of a piece of paper. The considerable contraction that occurs when aluminum is cooled from room temperature to 72 Kelvin makes this task challenging.

The configuration of FiST suggests that there will be some motion of the focal plane as it is cooled from room temperature to operating temperature. FiST is assembled at room temperature and the Kevlar strands that support the focal plane are tensioned to approximately $50 \mathrm{lbf}$. Upon cooling, the aluminum outer hub and inner cold block shrink. The Kevlar, however, will lengthen slightly upon cooling due to a slightly negative coefficient of thermal expansion in the axial direction. The rates at which each of the components of FiST shrink or expand will depend upon the cooling rate, the dimensions, and the material properties of that component.

As the aluminum parts of FiST expand and contract, the tension on the Kevlar strands will increase proportionally to the change in length of the strands. If the strands have all been tensioned to the same value, the force increase will be distributed evenly around the cold block, resulting in no change in angle of the focal plane relative to the outer hub. Uneven tension of the strands will likely result in a change in angle of the focal plane with respect to the outer hub as the assembly is cooled. This change in angle or the possible slipping of the strand fasteners in the through holes located in the outer hub is the source of concern. Also of concern is the tension profile of the Kevlar as the system is cooled. The outer hub of the FiST assembly is cooled to $250 \mathrm{~K}$ while the cold block to which the detectors are mounted is cooled to liquid nitrogen temperatures. If the inner 
hub cools rapidly and the outer hub cools slowly, will the tension in the Kevlar increase enough to damage or break the Kevlar strands? Will this in anyway effect the optical stability of the assembly? The Kevlar strands are angled about all three principal axes of the part. The compound angle of the Kevlar strands, the different geometry of the parts involved, and the differing thermal expansion coefficients make the prediction of tension increases in the strands anything but trivial.

The optical stability testing of FiST is a very complex and demanding procedure. Changes of about 0.001 inches on any part or joint of the test setup connected to FiST will result in at least 60 arc-seconds of error. Great care had to be taken to make an extremely robust and solid test setup. The test setup must also accurately simulate the back end of the SABER telescope to which the SABER FiST assembly is attached. To accomplish these goals the fixtures shown in Figures 3 \& 4 were designed.

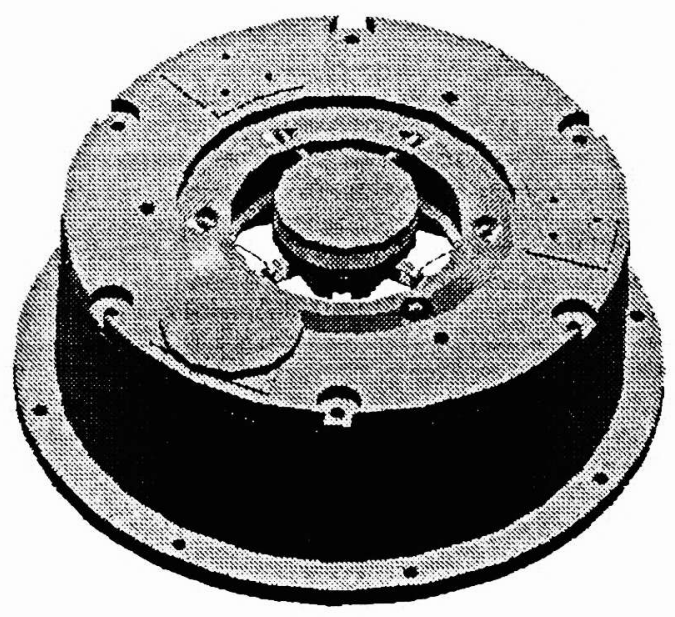

Figure 3. Optical stability test jig for FiST

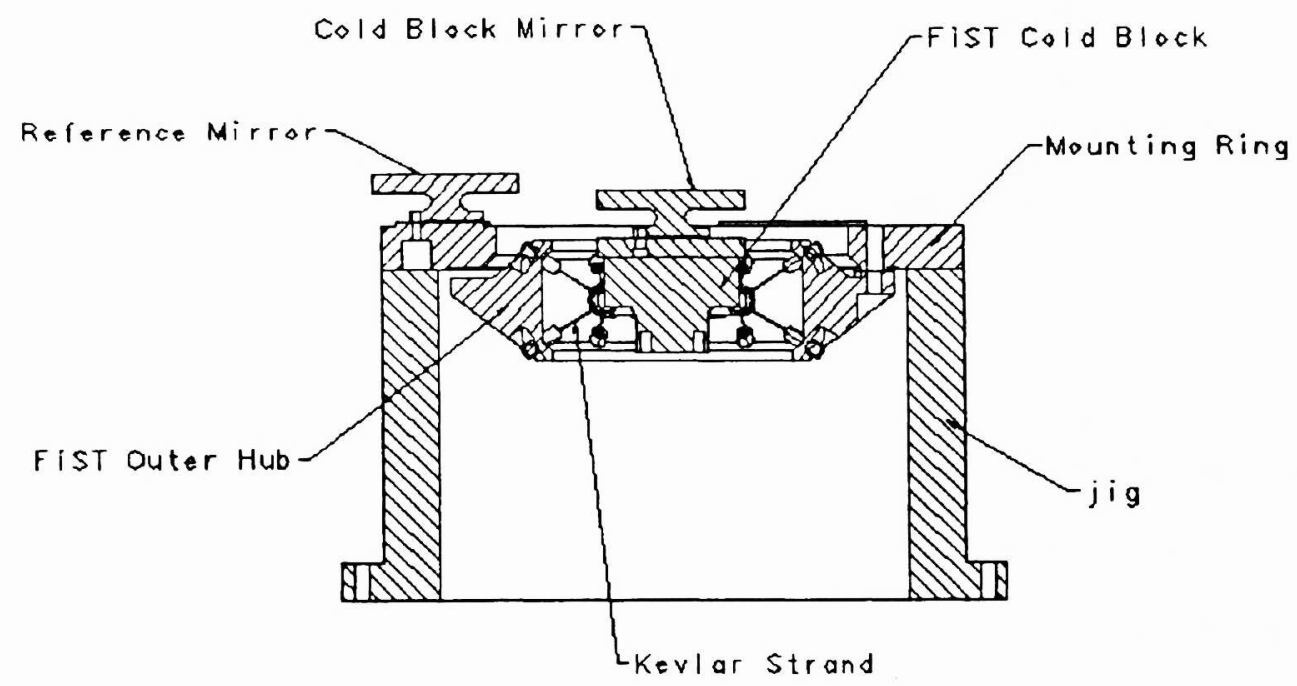

Figure 4. Optical stability test jig for FiST, cutaway view.

The setup consists of a mounting ring that is attached to a jig. The mounting ring simulates the back of the telescope while the jig simulates the spacecraft radiation environment. Optically flat mirrors were attached to both the cold block and to 
the mounting ring to measure the alignment of the focal plane relative to the telescope. FiST mounts to the mounting ring and is bolted in three places. Around each bolt hole a "pad" or small, flat, raised surface was machined. These pads were then lapped to achieve great flatness. This prevented any stresses in the mounting surfaces from causing erroneous angle changes. All critical bolted interfaces between FiST, the mirrors, and the mounting ring were mounted on these lapped pads. All parts were cryocycled after rough machining was performed. The parts were then machined to their final dimensions and cryo-cycled again.

The cold block of FiST was attached to the cold block of a liquid nitrogen dewar with a solder-less flexible thermal link. This provided a thermal path so that temperatures near $85 \mathrm{~K}$ could be reached. The Jig was isolated from the cold block of the test dewar so that a reasonable amount of heat would keep the outer hub of FiST at operational temperatures which are between $208 \mathrm{~K}$ in the cold case and $250 \mathrm{~K}$ in the hot case. This combination of isolation on the massive outer hub, and a thermal shunt on the small cold block of FiST, creates a cooling profile that is more harsh than FiST will ever experience during flight. The cooling profile of the inner and outer hubs was recorded during the first cool-down and is shown in Figure 5.

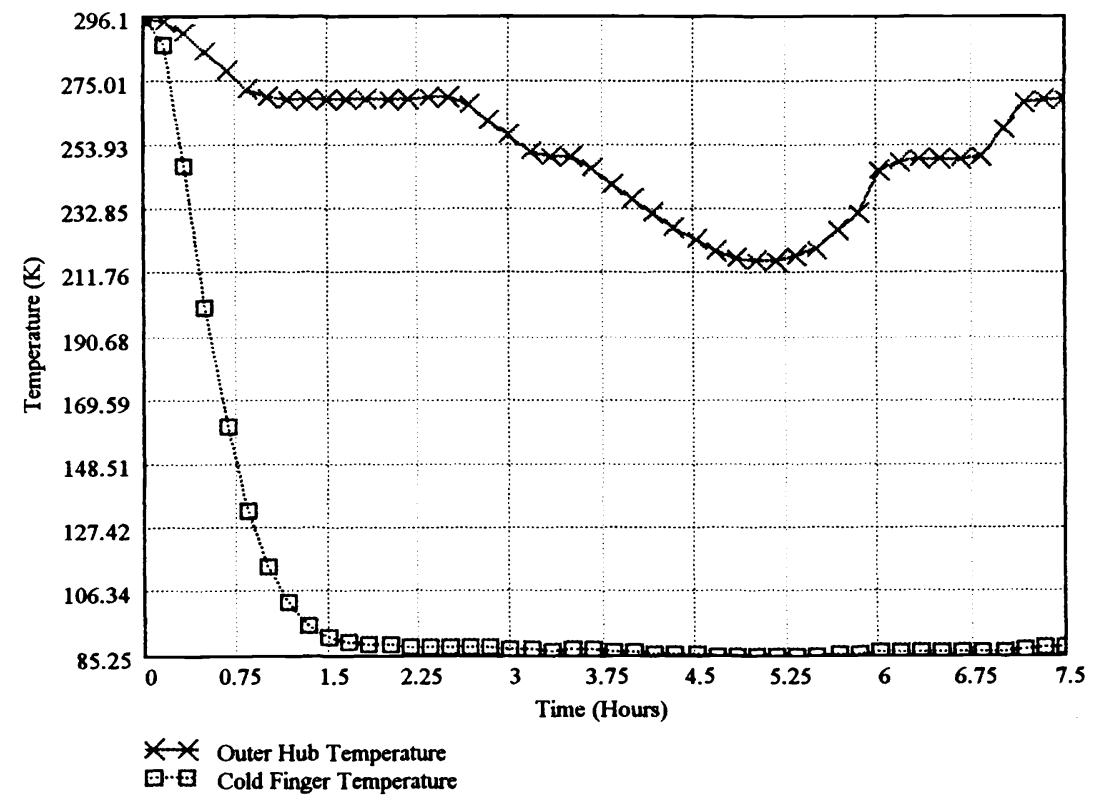

Figure 5. Temperature profile for optical stability test.

With the cooling profile known, the tension profile of the FiST Kevlar strands was modeled in MathCad. This model included all complex geometry and temperature variance of thermal expansion coefficients. Knowing the spring constant of the Kevlar, the force change experienced by the Kevlar during the stability testing was determined using the experimental temperature profile shown in Figure 5. Examination of the force change profile shown in Figure 6 shows that during initial cooling, when the cold block cools faster than the outer hub, a tension increase of approximately $25 \mathrm{lbf}$. is experienced. Conversely, during cold case operation a tension decrease (from the original tension of $50 \mathrm{lbf}$.) of approximately $25 \mathrm{lbf}$. is experienced. 


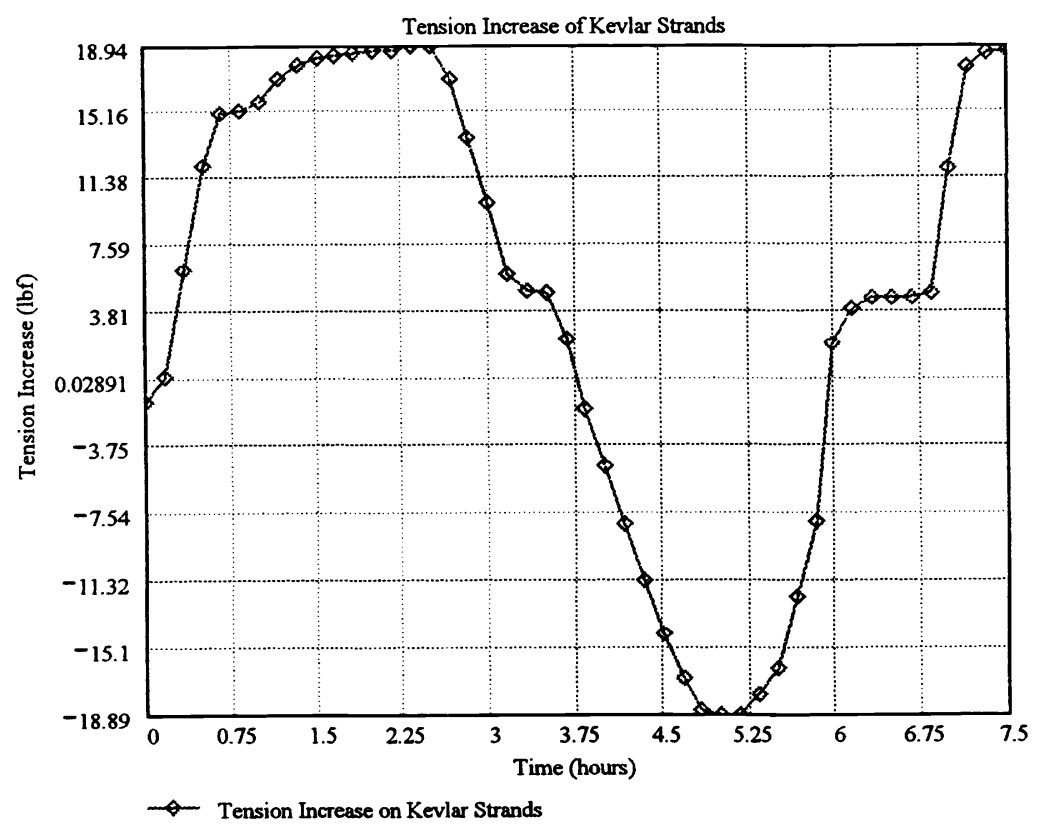

Figure 6. Tension profile of Kevlar strands during optical stability testing.

The Kevlar strands do experience a wide range of tension during the test, but as mentioned previously this will be much more severe than experienced during flight. The Kevlar was sized properly such that in the increase in tension still leaves a safety factor of 2.5 on the strand assembly

The relative angle between the mounting ring and the cold block of FiST was measured by placing a mirror on each of the lapped surfaces (see Figures $3 \& 4$ ). Two theodolites (similar to a rifle-scope that emits a cross-hair shaped beam of light) were used to measure the angle between the mirrors during the test.

Refer to Figure 7 for the following discussion. A theodolite was aligned with each mirror and the horizontal and vertical measurements of the theodolite aligned with the cold block (theodolite 2) mirror are recorded. A theodolite is aligned such that its cross-hair shaped beam of light is reflected back into itself off a mirror in the test setup. Since the theodolite has internal crosshairs (just like those on a rifle-scope), and can focus at infinity (where light rays are parallel) on the lighted cross hair it projects, it can be aligned perfectly perpendicular with the surface of a mirror by aligning the lighted cross-hairs with the internal ones. Then, without touching either theodolite, a large reference flat is brought into alignment (perfectly perpendicular) with the theodolite that is lined up with the reference mirror on the mounting ring. Now the theodolite that was aligned with the cold block is adjusted until it is aligned perfectly parallel to the axis of the reference flat, and its angular measurements are recorded. Note that angular measurements are only made with one theodolite (theodolite 2 ). The other theodolite is used merely for reference.

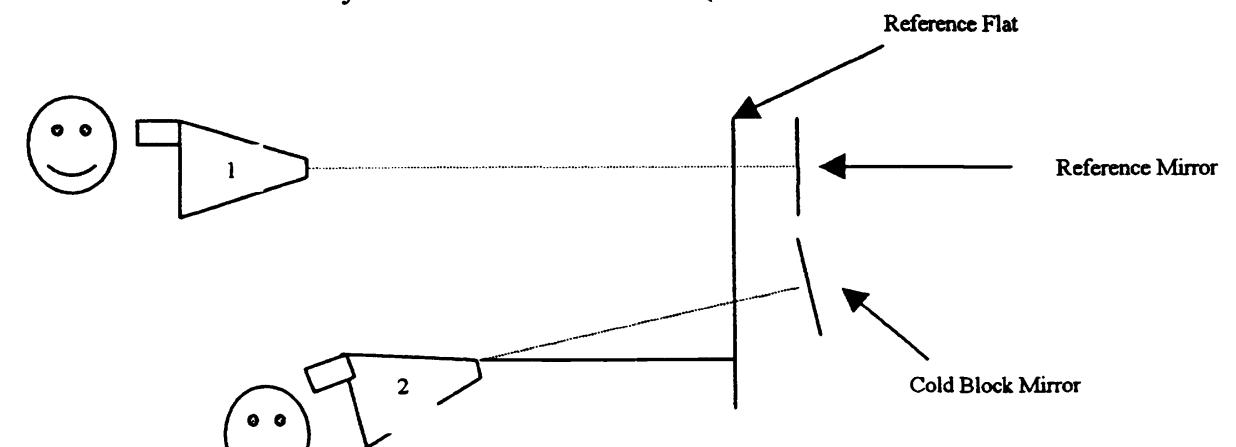

Figure 7. Theodolite alignment sketch. 
A few pictures of the actual test setup in the liquid nitrogen dewar and the theodolites used are shown in Figures 8 and 9 .

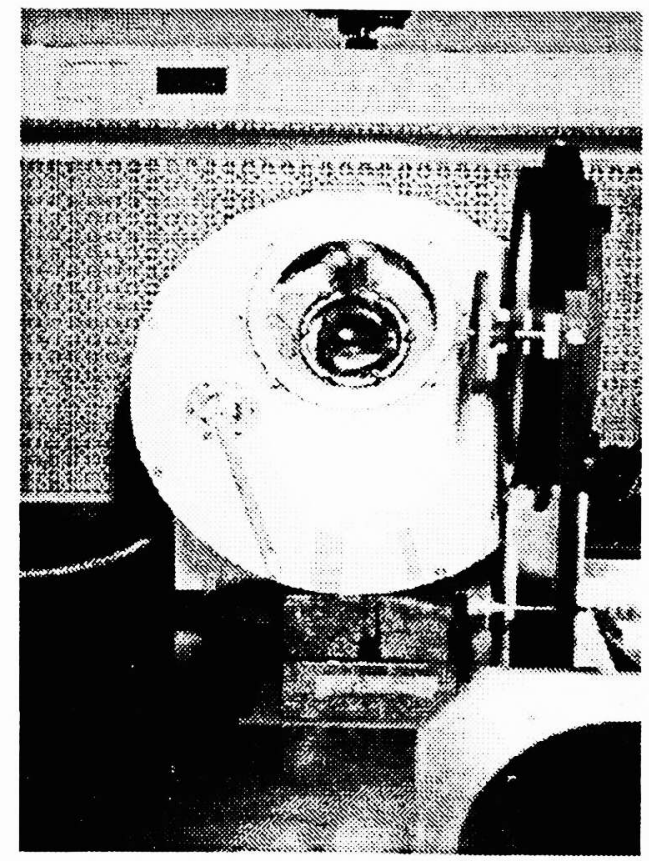

Figure 8. FiST assembly in test jig in liquid nitrogen dewar.

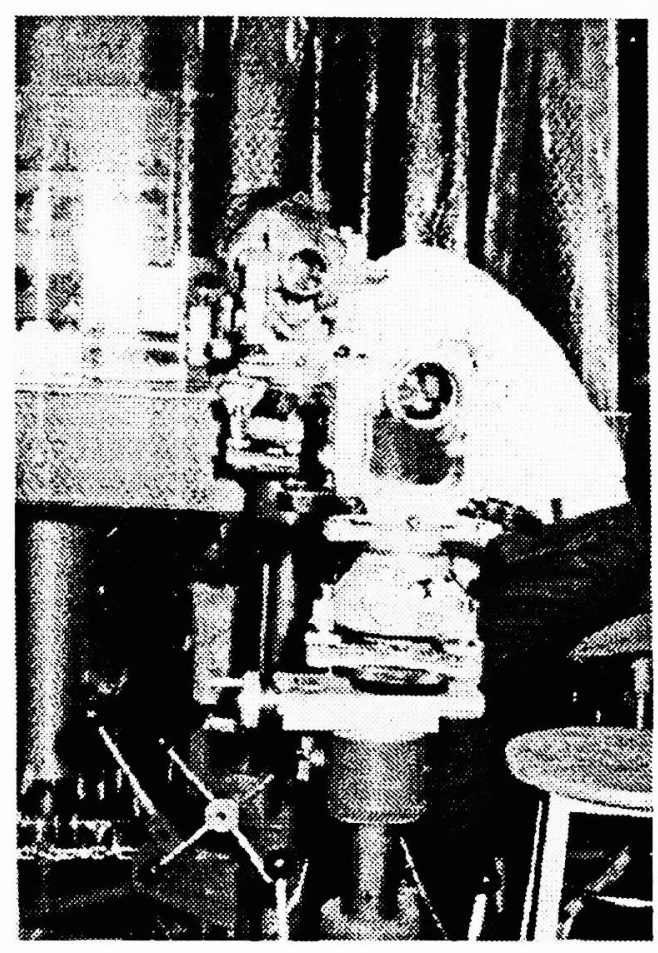

Figure 9. Theodolites used for optical stability testing. 
The test profile as shown in Figure 5 was to cycle from room temperature to the hot case operating scenario, then to the cold case operating scenario, back to the hot case and then finally to room temperature. Angle measurements were taken at each stopping point. The latest optical stability testing data can be found in Figures 10-15. Each plot shows the relative angle (or a component thereof) between the cold block and the outer hub of FiST. As is evident from these Figures, there are 3 traces, one labeled current test, one labeled previous test, and one labeled baseline measurement. This test was conducted two different times. The first time the data was a little discouraging as a considerable amount of movement was noticed. The source of the movement was determined to be the Kevlar fasteners slipping in the holes at their interface to the outer hub. This problem was solved by bonding the fasteners into their respective holes, and a new test was conducted. The baseline measurement in the plots represents the first measurement at temperature for the latest test.

Notice the angular change in the first few cycles. These changes can be attributed to stress relieving that occurs in the setup. Despite all efforts to avoid such problems, a few cycles must pass before repeatable performance is achieved. The majority of the movement seen during the first 10 cycles was determined to be the attachment of the FiST unit to the jig assembly and not movement of the cold finger with respect to the outer hub of FiST.

It can be seen by comparing the data taken at $250 \mathrm{~K}$ versus the data taken at $207 \mathrm{~K}$ that an angle change of about 20 arcseconds is realized between the cold finger and the outer hub of FiST. As predicted, any tension variation in the strands during assembly would cause an angle change during changes in operational temperatures. For the unit tested, if the telescope were to be initially aligned at $235 \mathrm{~K}$ a maximum change in angle of only 10 arc-seconds would be experienced. This is well within the tolerance of the optical stability specification. Note that once the initial stress relieving, the optical stability of FiST is extremely good. The fluctuations in the measurements shown in the charts are due to error in theodolite reading repeatability and not movement of the FiST assembly.

\section{$300 \mathrm{~K}$ Comparison \\ Vertical Angle}

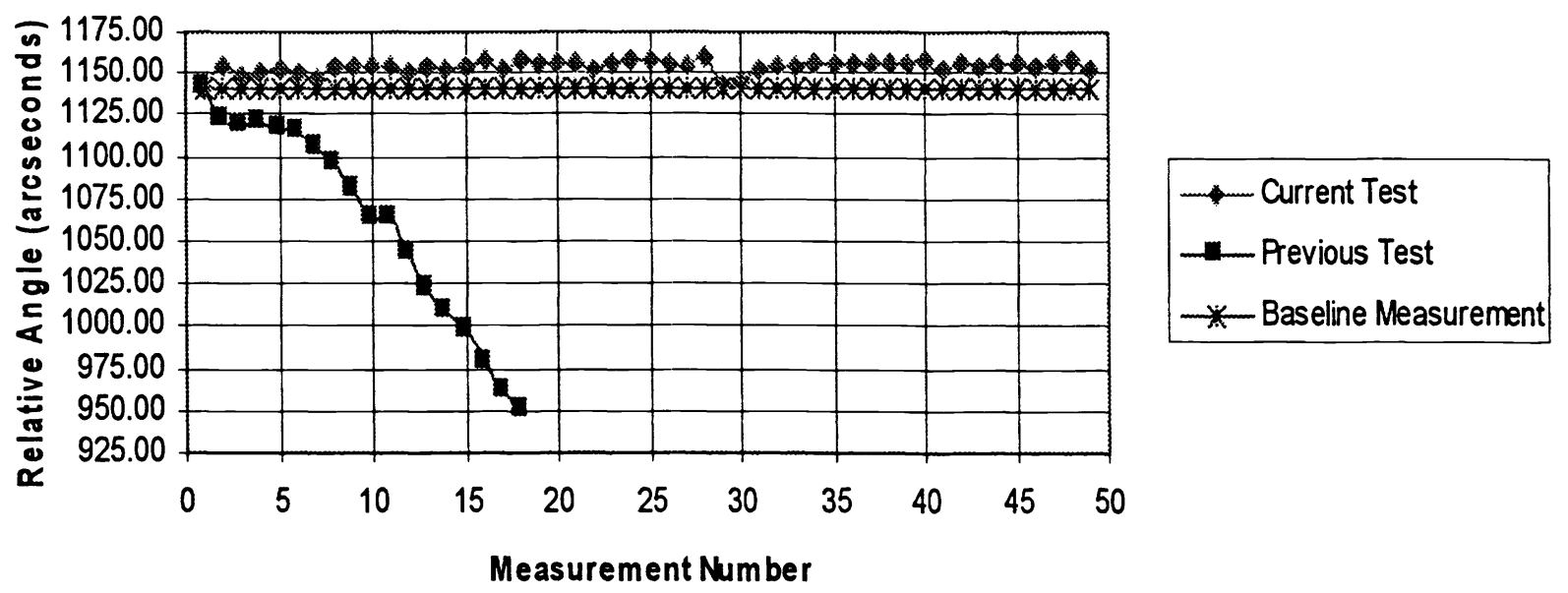

Figure 10. Test data: vertical component at room temperature 


\section{$300 \mathrm{~K}$ Comparison \\ Horizontal Angle}

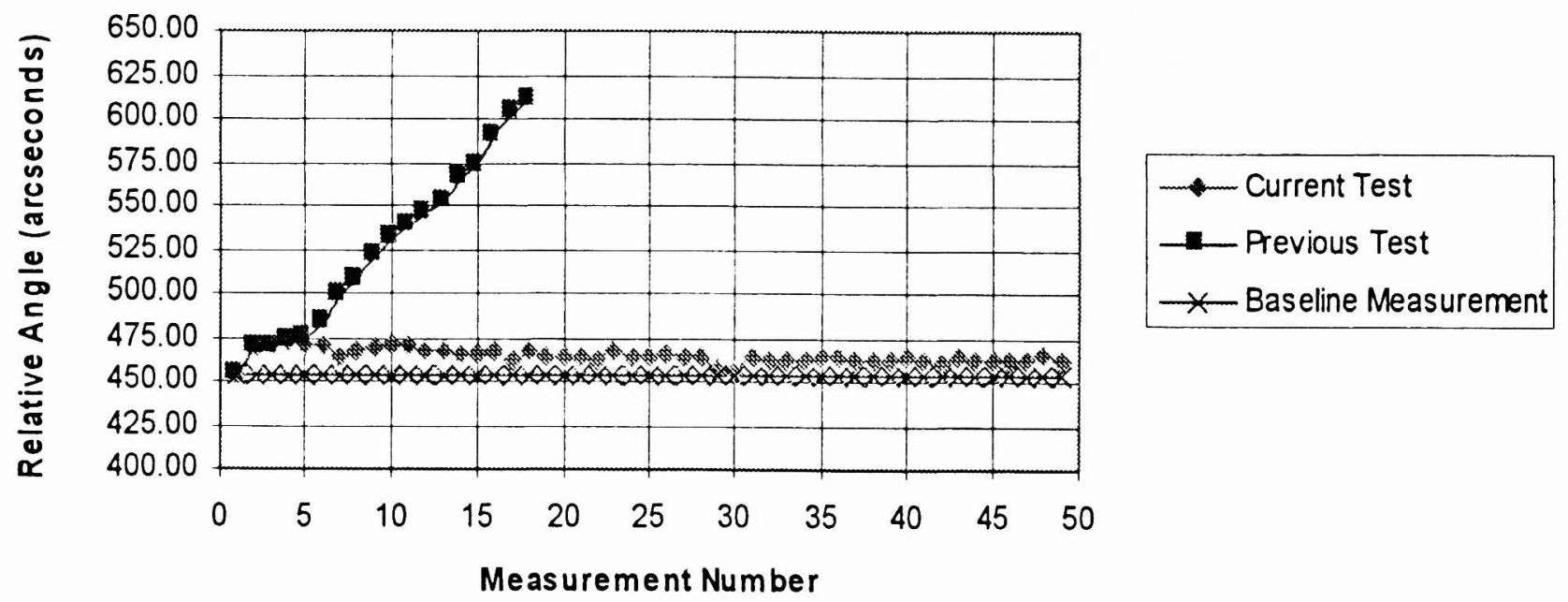

Figure 11. Test data: horizontal component at room temperature.

\section{K Comparison \\ Vertical}
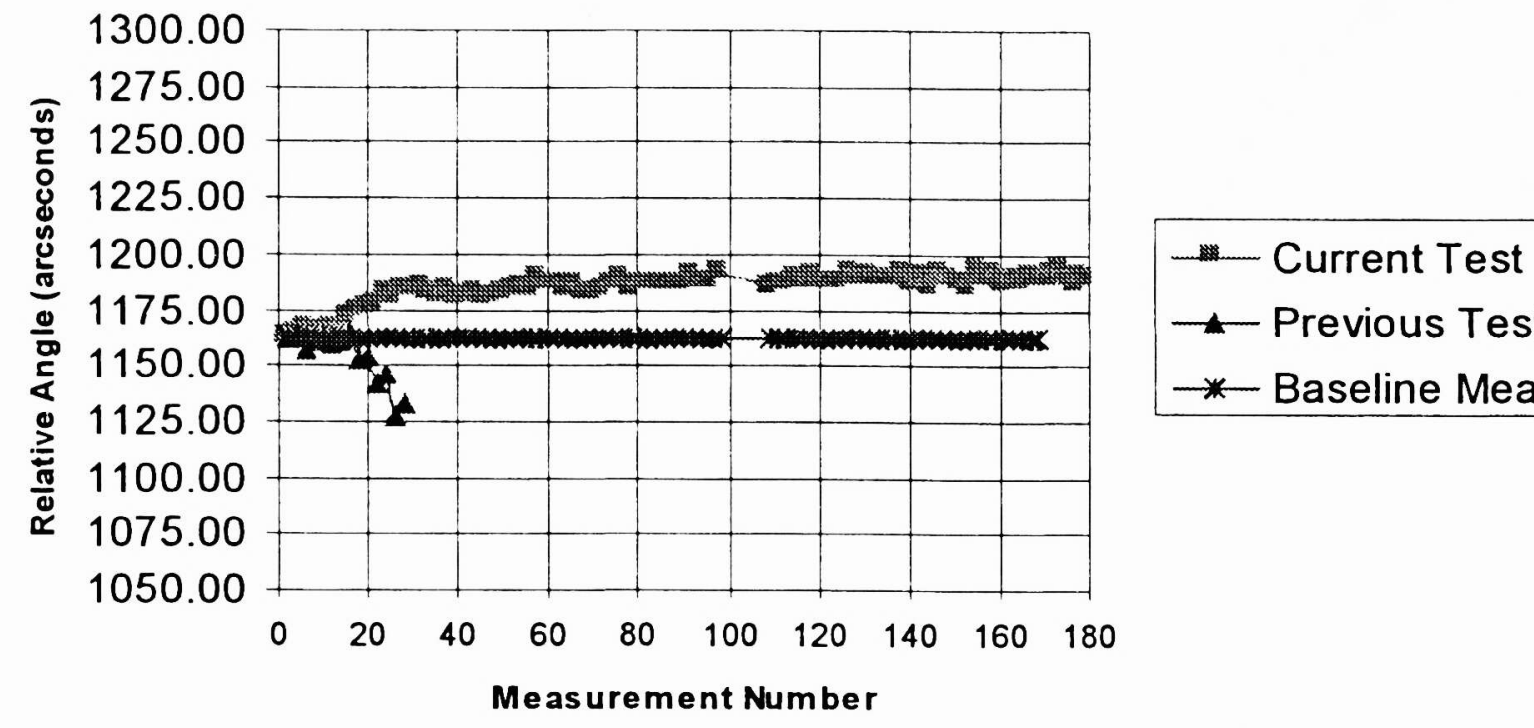

$\rightarrow$ Previous Test

* Baseline Measurement

Figure 12. Test data: vertical component, hot case. 


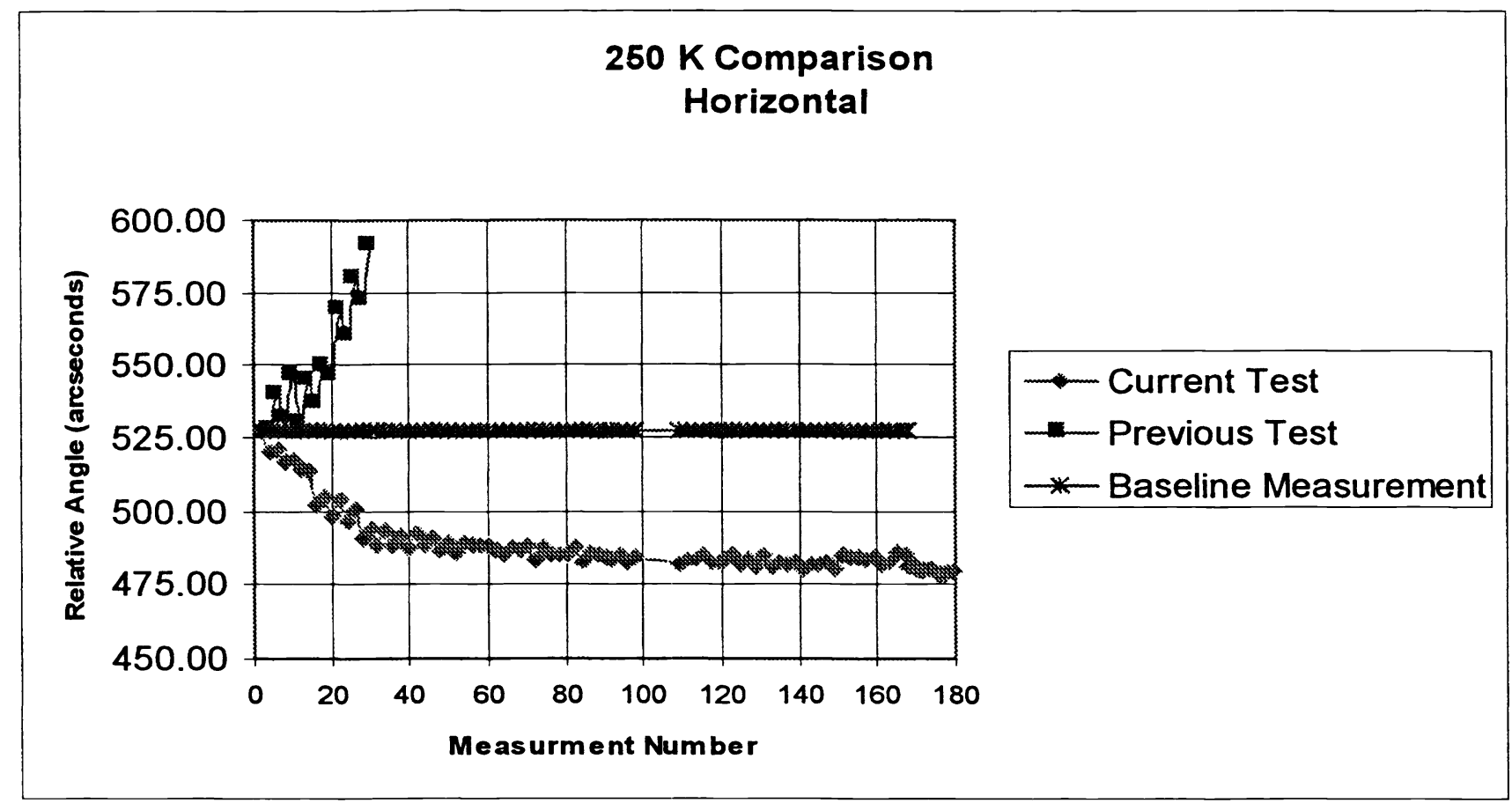

Figure 13. Test data: horizontal component, hot case.

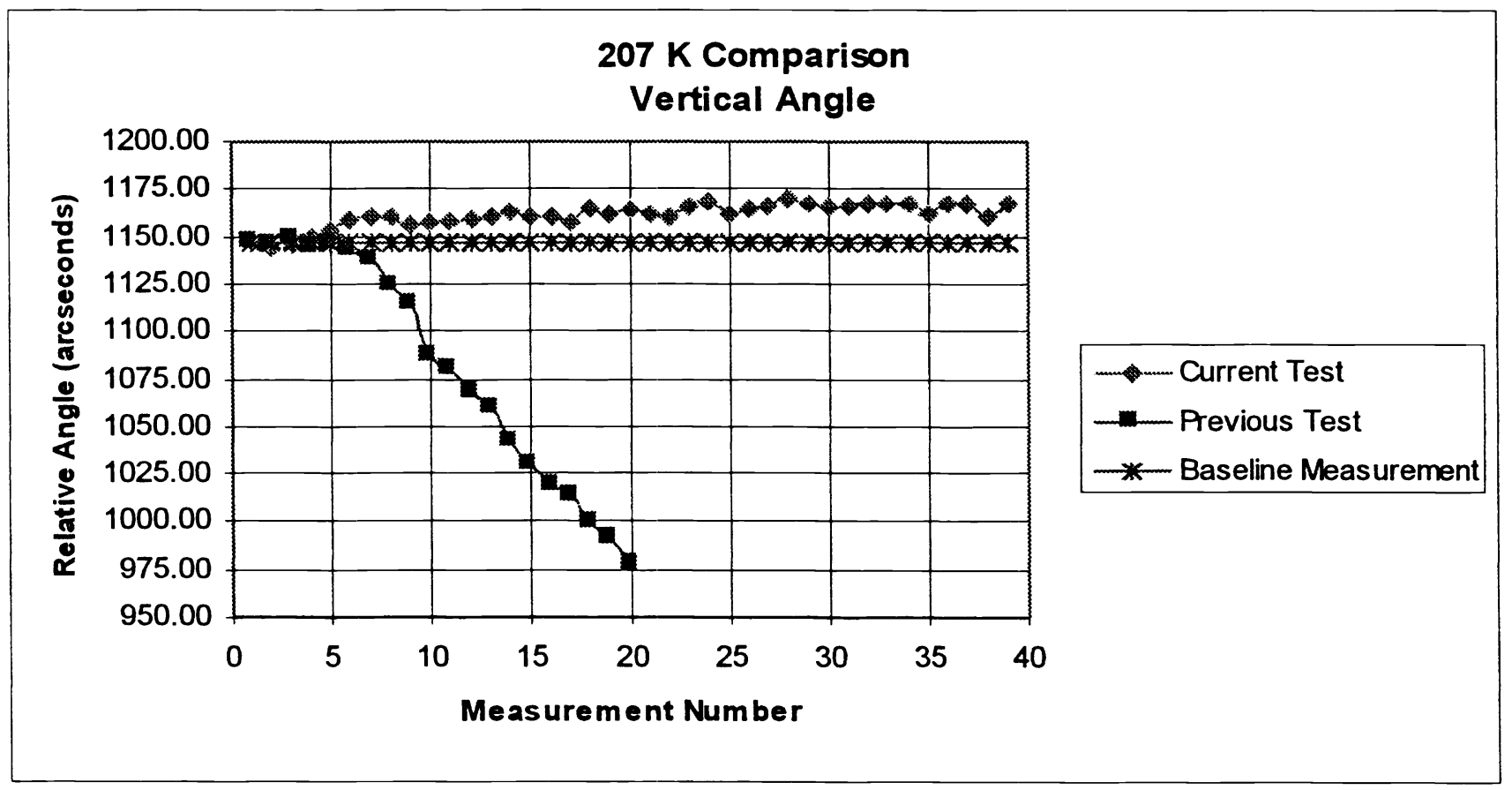

Figure 14. Test data: vertical component, cold case. 


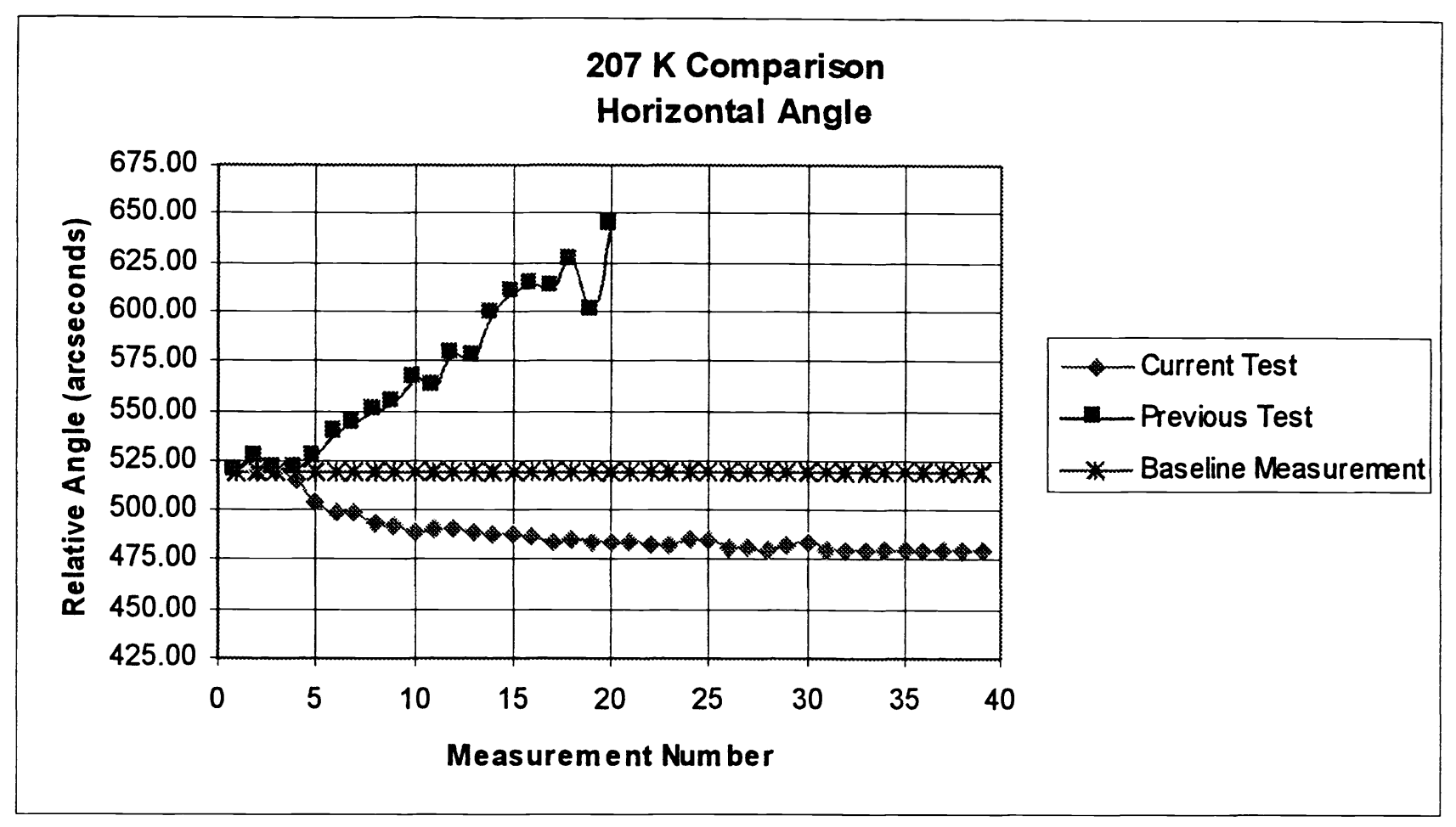

Figure 15. Test data: horizontal component, cold case.

With the successful completion of the thermal cycle stability tests, FiST was taken to the shaker table. At this point, because the test wasn't performed in a vacuum, a precision measurement machine called a coordinate measuring machine (CMM) was used to determine angular movement. Measurement repeatability on this machine is on the order of .0002 inches.

The FiST assembly was shaken about all three principal axes five times at a level of 10 grms. A measurement was taken at the completion of each shake to determine if any movement had taken place. Through the entire shake testing, no movement was measured by the CMM. This indicates that FiST is optically stable when subjected to vibrations similar to those experienced during launch.

\subsection{CONCLUSIONS}

The design of the FiST system for the SABER FPA is well on its way to completion. A successful breadboard model has been built and tested with very promising results. The prototype breadboard unit is mechanically robust having a first natural resonant frequency of nearly $800 \mathrm{~Hz}$ in all axes. Thermally, the FiST approach is second to none. Conduction parasitic heat loads have been reduced to a few milliwatts. FiST has been proven to be optically stable in the current configuration. The successful development of this technology will revolutionize the cryogenic support industry. With the capability of reducing parasitic heat loads onto cooled components without compromising the structural integrity of the system, FiST is a significant advancement in the ongoing effort to produce "smaller, better, faster, and cheaper" spacecraft.

\subsection{ACKNOWLEDGEMENTS}

Portions of this work were supported by a contract awarded to the Space Dynamics Lab by NASA Langley. Many thanks to them for the opportunity to develop this promising technology. 


\subsection{REFERENCES}

1. Burt, William W. 1995. SABER SRR, Volume I, 5 April 1995 to 6 April 1995. Logan, Utah State University, Space Dynamics Laboratory.

2. Jensen, Scott M., Batty, J. Clair, Mclain, David. Paper titled "Fiber Support Technology for thermal isolation and mechanical stability" given at SPIE conference in Denver, Colorado, 1996. 